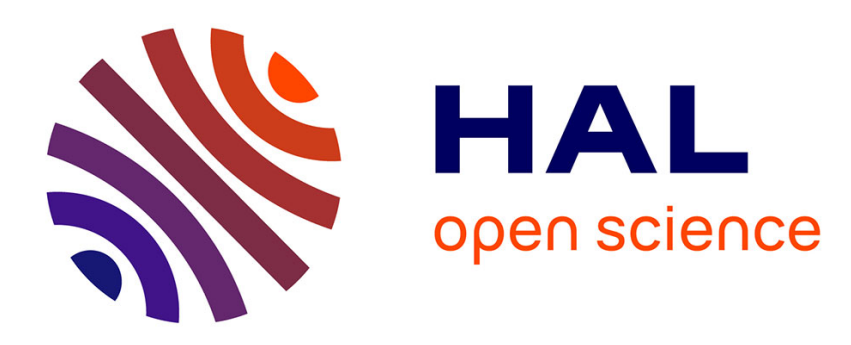

\title{
Détermination des paramètres d'un modulateur de vitesse à rassembleurs multiples
}

\author{
A. Noblet
}

\section{To cite this version:}

A. Noblet. Détermination des paramètres d'un modulateur de vitesse à rassembleurs multiples. Revue de Physique Appliquée, 1973, 8 (2), pp.197-206. 10.1051/rphysap:0197300802019700 . jpa-00243675

\section{HAL Id: jpa-00243675 https://hal.science/jpa-00243675}

Submitted on 1 Jan 1973

HAL is a multi-disciplinary open access archive for the deposit and dissemination of scientific research documents, whether they are published or not. The documents may come from teaching and research institutions in France or abroad, or from public or private research centers.
L'archive ouverte pluridisciplinaire HAL, est destinée au dépôt et à la diffusion de documents scientifiques de niveau recherche, publiés ou non, émanant des établissements d'enseignement et de recherche français ou étrangers, des laboratoires publics ou privés. 


\title{
DÉTERMINATION DES PARAMÈTRES D'UN MODULATEUR DE VITESSE A RASSEMBLEURS MULTIPLES
}

\author{
A. NOBLET \\ Laboratoire de Physique Electronique, Université Libre de Bruxelles, Bruxelles, Belgique
}

(Reçu le 30 octobre 1972, révisé le 26 janvier 1973)

\begin{abstract}
Résumé. - Lorsqu'on a recours à un dispositif de regroupement par modulation de vitesse pour produire des impulsions brèves de neutrons, il faut généralement utiliser un regroupeur multiple afin de maintenir l'amplitude de la tension modulante dans des limites raisonnables.

Nous avons établi les expressions théoriques qui permettent de déterminer les paramètres caractéristiques d'un modulateur à $n$ rassembleurs.

De plus, l'influence de différents paramètres sur le gain du système a été analysée afin de connaître les limites de variation admissibles.

Les expressions ainsi établies restent valables dans des limites telles qu'on couvre un très large domaine d'application.
\end{abstract}

\footnotetext{
Abstract. - When a buncher is used in order to get short pulses of neutrons, it is necessary to utilize a multiple gap buncher in order to maintain the bunching voltage within reasonable limits.

We have established the theoretical expressions necessary to calculate the characteristics parameters of a $n$-gap buncher.

Furthermore we have analysed in what way the variation of the various parameters modify the bunching ratio.

The final expressions remain valid within sufficient limits so as to cover a wide application range.
}

1. - Introduction. - De nombreuses recherches concernant par exemple la structure du noyau, la détermination des paramètres des réacteurs nucléaires et la spectrométrie des neutrons ont pu être menées à bien grâce au développement important que connaissent les sources pulsées de neutrons [1]. Généralement, les neutrons sont obtenus par bombardement d'une cible neutronigène à l'aide des protons ou de deutons. Par conséquent, ce sont essentiellement les accélérateurs de particules qui font l'objet de perfectionnements constants en vue de réduire la durée des impulsions de courant ionique à la cible, donc de réduire l'incertitude sur l'instant de départ des neutrons.

Le schéma le plus couramment adopté pour réaliser des impulsions courtes est le suivant : hachage du faisceau par déflection électro-statique suivi d'un regroupement des particules constitutives de l'impulsion de sortie du pulsateur [2].

Nous nous intéresserons plus spécialement ici à l'étude du regroupement par modulation de vitesse qui, du fait de sa relative facilité de mise en œuvre, a généralement supplanté les autres procédés de regroupement [3], [4].

Des résultats acquis, il apparaît, comme on peut d'ailleurs intuitivement le comprendre, que la profondeur de modulation, donc l'amplitude de la tension modulante, est directement liée à la distance disponible pour réaliser le regroupement.

Aussi, afin d'éviter la manipulation de tension haute fréquence trop élevée, on a assez rapidement imaginé d'utiliser des regroupeurs à deux ou trois rassembleurs [5].

De tels dispositifs permettent d'obtenir des impulsions neutroniques de l'ordre de $1 \mathrm{~ns}$ de largeur à mi-hauteur, par modulation de vitesse triple à $10 \mathrm{Mc} / \mathrm{s}$ sous une tension de $10 \mathrm{kV}$ au bout d'une distance de $1,5 \mathrm{~m}$ pour des deutons de $250 \mathrm{keV}$ [6].

Jusqu'à présent, cependant, il n'existe pas de théorie d'ensemble permettant de déterminer les paramètres d'un dispositif de regroupement à rassembleurs multiples et c'est là l'objet de la présente étude.

\section{Regroupement par modulation de vitesse. -} Diverses publications ont traité de ce sujet [4], [5]. Rappelons brièvement le principe de la modulation de vitesse : les ions traversent un rassembleur c'est-àdire un espace dans lequel règne un champ électrique parallèle à leur vitesse initiale $v_{0}$.

La phase d'entrée des particules est telle qu'on ralentit les premières alors que pour les dernières le signe de la variation de vitesse aura changé. Les particules traversent ensuite l'espace de glissement libre 
de champ où, sous l'effet des variations de vitesse imposées dans le rassembleur, a lieu le regroupement.

Ainsi par exemple, dans le cas d'une modulation à un seul rassembleur, nous pourrons écrire que la vitesse à la sortie de celui-ci aura pour expression

$$
v_{1}=v_{0}\left(1+\frac{U}{U_{0}} \sin \omega t_{1}\right)^{1 / 2}
$$

où $U_{0}$ est la tension d'accélération des ions,

$U$ est la tension de modulation,

$t_{1}$ est l'instant d'entrée dans le regroupeur.

Donc l'angle de transit jusqu'à la cible de l'ion de phase d'entrée $\theta_{1}=\omega t_{1}$ sera

$$
\theta_{\mathrm{c}}=\omega t_{\mathrm{c}}=\theta_{1}+\frac{\omega l}{v_{1}}
$$

où $l$ est la distance de glissement.

Si nous supposons $U$ plus petit que $U_{0}$, nous obtenons

$$
\theta_{\mathrm{c}}=\theta_{1}+L\left(1-\alpha \sin \theta_{1}\right)
$$

avec $L=\omega l / v_{0}$ et $\alpha=U / 2 U_{0}$.

Comme un regroupement impose que $\theta_{c}$ reste constant pour une variation de $\theta_{1}$, nous devons écrire :

$$
\frac{\mathrm{d} \theta_{\mathrm{c}}}{\mathrm{d} \theta_{1}}=1-\alpha L \cos \theta_{1}=0
$$

ce qui en optimisant la valeur obtenue pour $L$ nous conduit à une valeur particulière de $\theta_{1}$ qui est la phase de l'ion autour duquel s'effectue le regroupement

$$
\frac{\mathrm{d} L}{\mathrm{~d} \theta_{1}}=0 \quad \text { donc } \quad \sin \theta_{1}=0
$$

et de ce fait $L_{\mathrm{opt}}=1 / \alpha$.

Par conséquent, un regroupeur simple travaillant dans les mêmes conditions que le regroupeur triple cité plus haut, c'est-à-dire $f=10^{7} \mathrm{c} / \mathrm{s}, l=1,5 \mathrm{~m}$, $U_{0}=250 \mathrm{kV}$, nécessitera l'emploi d'une tension de modulation de l'ordre de $25 \mathrm{kV}$.

3. Modulation de vitesse à rassembleurs multiples. - Le schéma de principe d'un tel regroupeur est donné en figure 1. Différentes hypothèses et remarques sont à faire.

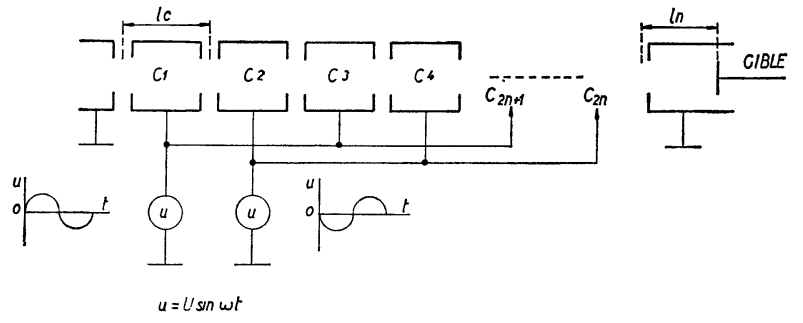

Fig. 1. - Schéma de principe d'un regroupeur à $n$ rassembleurs.
1) Nous considérons chaque rassembleur comme étant infiniment court donc la grandeur de l'impulsion de vitesse sera uniquement fonction de l'instant d'entrée de la particule dans le rassembleur.

Pour rencontrer pratiquement cette hypothèse, il faut que :

$$
\tau \ll T
$$

avec $\tau$ : temps de transit des ions dans le rassembleur, et $T$ : période de la tension de modulation.

2) Un générateur de tension sinusoïdale fournit deux tensions symétriques. La tension de modulation est de la forme

$$
u=U \sin \omega t
$$

dans le premier et le dernier rassembleur. Elle a une valeur double dans les autres rassembleurs.

3) La première et la dernière cellule sont maintenues au potentiel de la masse, ceci pour des raisons de commodité pratique.

4) Le temps de transit sans modulation des ions dans les cellules, sauf la dernière vaut $T / 2$.

Ceci est nécessaire afin que les ions d'une même impulsion soient influencés dans chaque rassembleur par un champ électrique ayant toujours le même signe.

Nous aurons donc :

$$
\frac{\omega l_{\mathrm{c}}}{v_{0}}=\pi
$$

où $l_{\mathrm{c}}$ est la longueur d'une cellule,

$v_{0}$ est la vitesse initiale des ions

et $\omega$ est la pulsation de la tension de modulation.

5) On suppose que le faisceau à l'entrée du regroupeur est monocinétique et cylindrique.

Pratiquement, le regroupement s'effectue après un tri en énergie des particules du faisceau.

6) On négligera les effets dus à la charge d'espace.

Les paramètres caractéristiques à déterminer sont :

- la distance minimum de regroupement,

- la phase d'entrée de l'ion central autour duquel s'effectue le regroupement,

- le gain du regroupeur ou facteur de regroupement donnant le rapport de la durée de l'impulsion d'entrée à celle de l'impulsion cible.

Nous mènerons le calcul de la façon suivante : connaissant la vitesse de l'ion de phase d'entrée $\theta_{1}$ à la sortie de chaque rassembleur, on peut déterminer son instant d'arrivée à la cible

$$
t_{\mathrm{c} n}=t_{\mathrm{c} n}\left(t_{1}, l_{n}\right) \quad \text { avec } \quad \theta_{1}=\omega t_{1} .
$$


La longueur $l_{n}$, distance séparant le dernier rassembleur de la cible, apparaît comme paramètre dans cette expression.

Posons :

$$
L_{n}=\frac{\omega l_{n}}{v_{0}}
$$

on pourra écrire

$$
\theta_{\mathrm{c} n}=\theta_{\mathrm{cn}}\left(\theta_{1}, L_{n}\right) \quad \text { avec } \quad \theta_{\mathrm{c} n}=\omega t_{\mathrm{c} n} .
$$

En écrivant la loi de conservation de la charge

$$
I_{0} \mathrm{~d} t_{1}=I_{\mathrm{c}} \mathrm{d} t_{\mathrm{c}}
$$

ou encore

$$
I_{0} \mathrm{~d} \theta_{1}=I_{\mathrm{c}} \mathrm{d} \theta_{\mathrm{c} n}
$$

$I_{0}$ étant le courant à l'entrée du regroupeur et $I_{\mathrm{c}}$ le courant cible, on voit qu'on aura regroupement pour

$$
\frac{\mathrm{d} \theta_{\mathrm{cn}}}{\mathrm{d} \theta_{1}}=0
$$

Cette condition établit une relation entre $\theta_{1}$ et $L_{n}$ telle qu'il existe plusieurs couples de valeurs $\theta_{1}$, $L_{n}$ y répondant.

Parmi ces couples, on cherchera celui qui conduit à une longueur $l_{n}$ minimum c'est-à-dire tel que

$$
\frac{\mathrm{d} L_{n}}{\mathrm{~d} \theta_{1}}=0
$$

ce qui introduira une valeur particulière $\theta_{10}$ de $\theta_{1}$ qui est la phase d'entrée de l'ion central.
La distance de glissement minimum sera évidemment donnée par

$$
L_{n \text { opt }}=L_{n}\left(\theta_{10}\right) .
$$

Pour calculer le gain $G \mathrm{du}$ regroupeur, on part de l'expression

$$
\Delta \theta_{\mathrm{c} n}=\frac{\mathrm{d} \theta_{\mathrm{c} n}}{\mathrm{~d} \theta_{1}} \Delta \theta_{1}+\frac{\mathrm{d}^{2} \theta_{\mathrm{cn}}}{\mathrm{d} \theta_{1}^{2}} \frac{\Delta \theta_{1}^{2}}{2}+\frac{\mathrm{d}^{3} \theta_{\mathrm{c} n}}{\mathrm{~d} \theta_{1}^{3}} \frac{\Delta \theta_{1}^{3}}{6}+\cdots
$$

qu'il faut apprécier autour de $\theta_{1}=\theta_{10}$.

Dans cette expression $\Delta \theta_{c n}$ et $\Delta \theta_{1}$ représentent respectivement la demi-largeur à la cible et la demilargeur à l'entrée du regroupeur. Comme les calculs précédents imposent que

$$
\left(\frac{\mathrm{d} \theta_{\mathrm{cn}}}{\mathrm{d} \theta_{1}}\right)_{\theta_{1}=\theta_{10}}=\left(\frac{\mathrm{d}^{2} \theta_{\mathrm{cn}}}{\mathrm{d} \theta_{1}^{2}}\right)_{\theta_{1}=\theta_{10}}=0
$$

il ne subsistera dans l'expression du gain que la dérivée troisième, donc

$$
G=\frac{\Delta \theta_{1}}{\Delta \theta_{c n}}=\frac{6}{\Delta \theta_{1}^{2} \frac{\mathrm{d}^{3} \theta_{\mathrm{cn}}}{\mathrm{d} \theta_{1}^{3}}}
$$

4. Calcul des paramètres d'un regroupeur multiple. - En se reportant au schéma de principe du regroupeur (Fig. 1), on pourra écrire l'expression de la vitesse de l'ion de phase d'entrée $\theta_{1}$ à la sortie de chaque rassembleur

$$
\begin{aligned}
& v_{1}=v_{0}\left(1+2 \alpha \sin \theta_{1}\right)^{1 / 2} \\
& v_{2}=v_{0}\left(1+2 \alpha \sin \theta_{1}-4 \alpha \sin \theta_{2}\right)^{1 / 2} \\
& v_{3}=v_{0}\left(1+2 \alpha \sin \theta_{1}-4 \alpha \sin \theta_{2}+4 \alpha \sin \theta_{3}\right)^{1 / 2} \\
& \ldots \\
& v_{n}=v_{0}\left[1+2 \alpha\left(\sin \theta_{1}+(-1)^{n-1} \sin \theta_{n}+2 \sum_{i=2}^{n-1}(-1)^{i-1} \sin \theta_{i}\right)\right]^{1 / 2}
\end{aligned}
$$

$v_{n}$ est la vitesse à la sortie du $n$-ième rassembleur $(n>2), \alpha, U$ et $U_{0}$ ont la même signification que précédemment et $\theta_{i}$ est la phase d'entrée de l'ion dans le $i$-ième rassembleur.

On peut alors déterminer l'angle de transit de cet ion jusqu'à la cible :

$$
\theta_{\mathrm{c} n}=\theta_{n}+\frac{\omega l_{n}}{v_{n}}=\theta_{n}+L_{n} \frac{v_{0}}{v_{n}}
$$

Remplaçant $v_{n}$ par sa valeur éq. (1), et tenant compte du fait que $2 \alpha$ est beaucoup plus petit que 1 , ceci nous donne :

$$
\theta_{\mathrm{c} n}=\theta_{n}+L_{n}\left[1-\alpha F\left(\theta_{1}, \theta_{i}, \theta_{n}\right)+\frac{3 \alpha^{2}}{2} F^{2}\left(\theta_{1}, \theta_{i}, \theta_{n}\right)+\cdots\right]
$$

avec

$$
F\left(\theta_{1}, \theta_{i}, \theta_{n}\right)=\sin \theta_{1}+(-1)^{n-1} \sin \theta_{n}+2 \sum_{i=2}^{n-1}(-1)^{i-1} \sin \theta_{i}
$$


Or nous avons vu que pour calculer les paramètres du regroupeur, il fallait partir d'une expression donnant $\theta_{c n}$ en fonction de $\theta_{1}$. Il faudra donc exprimer dans l'éq. (2), $\theta_{n}$ et $\theta_{i}$ en fonction de la phase d'entrée.

La longueur des cellules étant constante, nous avons

ou encore

$$
\theta_{i}=\theta_{i-1}+\frac{\pi v_{0}}{v_{i-1}} \quad i=2,3,4, \ldots, n
$$

$$
\theta_{i}=\theta_{i-1}+\pi\left[1-\alpha H\left(\theta_{1}, \theta_{j}\right)+\frac{3 \alpha^{2}}{2} H^{2}\left(\theta_{1}, \theta_{j}\right)+\cdots\right]
$$

avec

$$
H\left(\theta_{1}, \theta_{j}\right)=\sin \theta_{1}+2 \sum_{j=2}^{i-1}(-1)^{j-1} \sin \theta_{j}
$$

Ces expressions nous permettent de déterminer une forme approchée de $\sin \theta_{i}$ valable pour

$$
\alpha \leqslant \frac{0,1}{\pi(n-1)^{2}}
$$

Ceci étant établi, nous pouvons à présent exprimer $\theta_{c n}$ en fonction de $\theta_{1}$, ce qui, tout calcul fait, nous donne

$\theta_{n}=\theta_{1}+(n-1) \pi-(n-1)^{2} \alpha \pi \sin \theta_{1}+\alpha^{2} \pi^{2} \sin 2 \theta_{1} \sum_{i=2}^{n-1}(n-i)(i-1)^{2}+$

$$
+\frac{3 \alpha^{2}}{2} \pi \sin ^{2} \theta_{1}\left[n-1+4 \sum_{i=2}^{n-1} i(i-1)\right]
$$

et

$$
\begin{aligned}
\theta_{\mathrm{c} n}=\theta_{1}+(n-1) \pi+L_{n}-[(n-1) \pi & \left.+2 L_{n}\right](n-1) \alpha \sin \theta_{1} \\
& +\frac{\alpha^{2} \pi}{6}(n-1) \sin 2 \theta_{1}\left\{\frac{n \pi}{2}[n(n-3)+2]+L_{n}[2 n(n-2)+3]\right\} \\
& +\alpha^{2}(n-1) \sin ^{2} \theta_{1}\left\{\frac{\pi}{2}[4 n(n-2)+3]+6 L_{n}(n-1)\right\} .
\end{aligned}
$$

A titre d'exemple, nous présentons en figure 2 la variation de $\theta_{\mathrm{cn}}$ avec $\theta_{1}$, pour une modulation quintuple $(n=5)$. La valeur de $\alpha$ choisie correspond à la limite de validité tirée de l'éq. (5).
La courbe en traits pointillés (.) représente la solution théorique exacte calculée, par récurrence à l'ordinateur, à partir des expressions de départ à savoir pour la particule de phase d'entrée $\theta_{1}$ :

$$
\begin{aligned}
& v_{1}=v_{0}\left(1+2 \alpha \sin \theta_{1}\right)^{1 / 2} \\
& \theta_{2}=\theta_{1}+\pi \frac{v_{0}}{v_{1}} \\
& v_{2}=v_{0}\left(1+2 \alpha \sin \theta_{1}-4 \alpha \sin \theta_{2}\right)^{1 / 2} \\
& \theta_{3}=\theta_{2}+\pi \frac{v_{0}}{v_{2}} \\
& \vdots \\
& v_{n}=v_{0}\left\{1+2 \alpha\left[\sin \theta_{1}+(-1)^{n-1} \sin \theta_{n}+2 \sum_{i=2}^{n-1}(-1)^{i-1} \sin \theta_{i}\right]\right\}^{1 / 2} \\
& \theta_{\mathrm{c} n}=\theta_{n}+L_{n} \frac{0}{v_{n}} .
\end{aligned}
$$


La courbe en traits croix $(+)$ a été obtenue à partir des valeurs approchées tirées de (6).

On voit que la concordance entre ces courbes est excellente. Par ailleurs, l'examen de cette figure nous permet de conclure que la stabilité de la phase d'entrée de l'ion central n'est pas critique. Suivant le dérou- lement du calcul décrit plus haut, nous pouvons à présent calculer la phase d'entrée de cet ion central et la distance minimum de regroupement, ce qui nous donnera dans les approximations faites éq. (5)

$$
\sin \theta_{10} \simeq \theta_{10}=-6(n-1) \alpha
$$

et

$L_{n}=\frac{1-(n-1)^{2} \alpha \pi \cos \theta_{10}+\frac{n-1}{2}[4 n(n-2)+3] \alpha^{2} \pi \sin 2 \theta_{10}+\frac{n(n-1)}{6}[n(n-3)+2] \alpha^{2} \pi^{2} \cos 2 \theta_{10}}{\alpha\left\{2(n-1) \cos \theta_{10}-6(n-1)^{2} \alpha \sin 2 \theta_{10}-\frac{n-1}{3}[2 n(n-2)+3] \alpha \pi \cos 2 \theta_{10}\right\}}$.

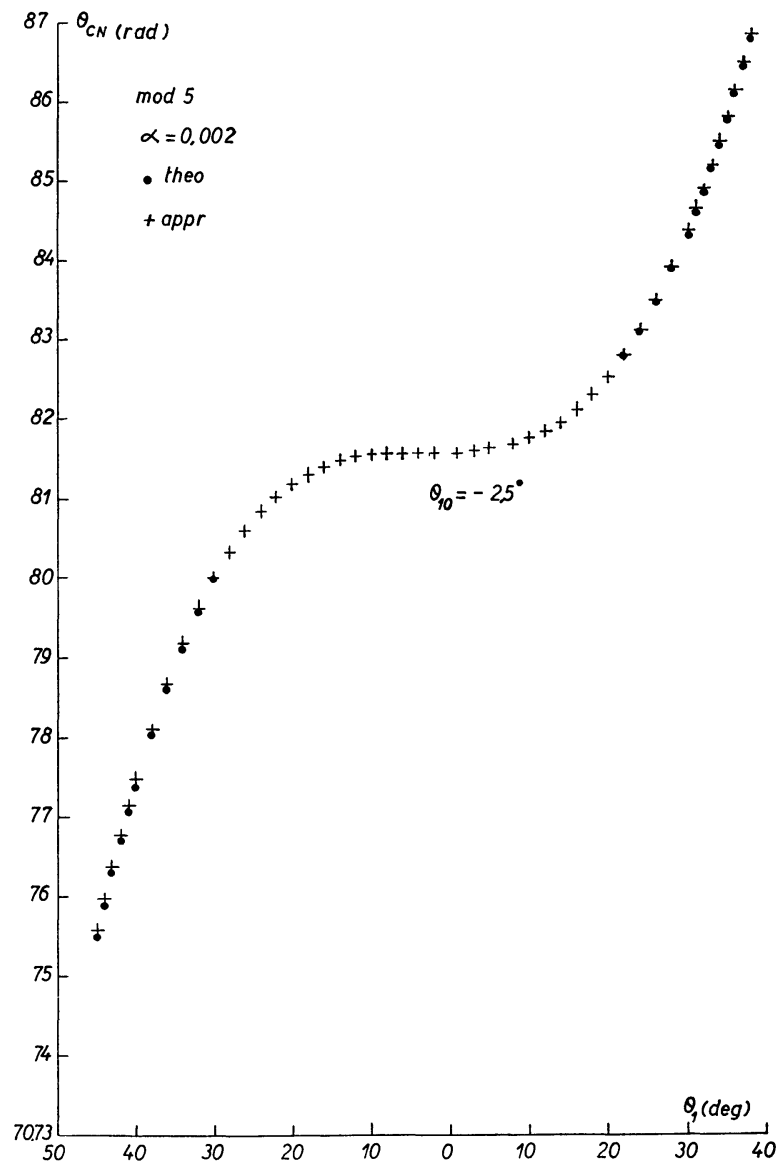

FIG. 2. - Variation de l'angle de transit $\theta_{\mathrm{e} n}$ en fonction de la phase d'entrée $\theta_{1}$.

Enfin il est également possible de déterminer le facteur de regroupement d'un modulateur multiple.

Nous savons que

$$
G=6\left[\Delta \theta_{1}^{2}\left(\frac{\mathrm{d}^{3} \theta_{\mathrm{cn}}}{\mathrm{d} \theta_{1}^{3}}\right)_{\theta_{1}=\theta_{10}}\right]^{-1} .
$$

Dans l'expression de la dérivée troisième de $\theta_{\mathrm{c} n}$, nous utiliserons une expression simplifiée de $L_{n}$ à savoir $L_{n}=\frac{1-(n-1)^{2} \alpha \pi}{\alpha\left\{2(n-1)-\frac{n-1}{3}[2 n(n-2)+3] \alpha \pi\right\}}$.
Dans ces conditions, nous aurons finalement

$$
\begin{aligned}
\left(\frac{\mathrm{d}^{3} \theta_{\mathrm{cn}}}{\mathrm{d} \theta_{1}^{3}}\right)_{\theta_{1}=\theta_{10}}= & (n-1)^{2} \alpha \pi+6 K(n, \alpha)- \\
& -4 \alpha \pi[2 n(n-2)+3] K(n, \alpha)
\end{aligned}
$$

avec

$$
K(n, \alpha)=\frac{1-(n-1)^{2} \alpha \pi}{6-[2 n(n-2)+3] \alpha \pi} .
$$

La figure 3 nous renseigne sur la validité des résultats obtenus par ce calcul.

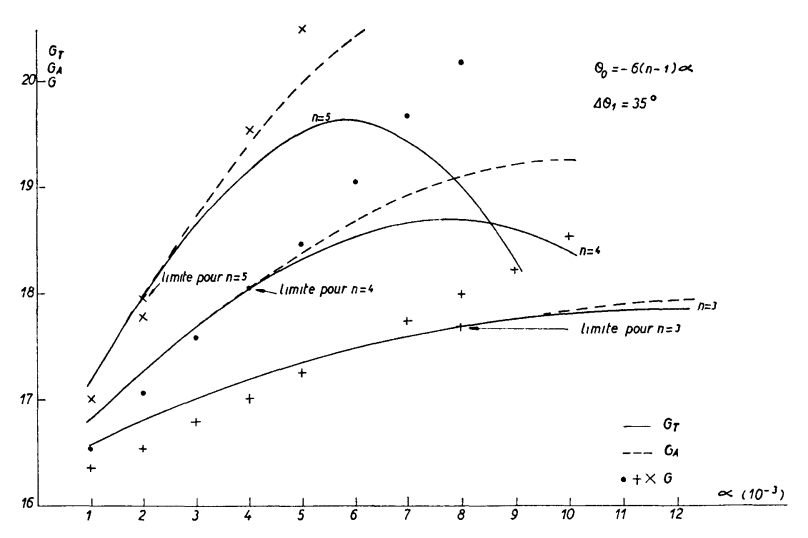

FIG. 3. - Variation du gain avec la profondeur de modulation.

Nous y avons représenté la variation du gain en fonction de $\alpha$, pour différents types de modulation. Le gain a été déterminé de trois manières distinctes ce qui explique la présence de trois courbes pour chaque type de modulation.

Les valeurs de $G_{\mathrm{T}}$ sont déterminées à l'aide d'un programme qui pour chaque valeur de $\alpha$ calcule la phase d'entrée de l'ion central $\theta_{10}$, la distance optimum de regroupement $L_{n}$ et enfin le rapport de la largeur d'impulsion à l'entrée $\Delta \theta_{1}$, centrée sur $\theta_{10}$ à la largeur d'impulsion correspondante à la cible $\Delta \theta_{c n}$ et ce à partir des expressions théoriques exactes de $\theta_{\mathrm{cn}}$ et $\theta_{i}$. 
Les valeurs de $G_{\mathrm{A}}$ sont calculées de la même façon que $G_{\mathrm{T}}$ mais à partir de l'expression approchée de $\theta_{c n}$ établie ci-dessus éq. (6).

Les valeurs de $G$ enfin correspondent au gain calculé directement à l'aide de la formule (7).

Ce graphique nous indique que cette dernière formule permet de déterminer le gain avec un écart de quelques $\%$ par rapport à la valeur théorique $G_{\mathrm{T}}$ pour autant qu'on ne s'écarte pas exagérément de la limite de validité des expressions établies.

Remarquons que dans les mêmes limites, le gain n'est pas fortement lié au type de modulation. C'est ainsi que le modulateur simple aura un gain à peu près constant de 16,5 pour $\Delta \theta_{1}=35^{\circ}$ et $\alpha=10^{-3}$ à $10^{-2}$, il augmentera de $3 \%$ environ pour $\alpha=10^{-1}$.

5. Etude du gain. - Il est intéressant de connaître la façon dont le gain du regroupeur est lié aux différents paramètres qui apparaissent dans son expression. On pourra de cette façon déterminer, par exemple, la stabilité de fréquence à exiger du générateur afin de maintenir les variations de la largeur d'impulsions cible dans des limites données.

Cette largeur d'impulsions sera également fonction de la vitesse des ions à l'entrée du regroupeur ainsi que de la stabilité de l'amplitude de la tension de modulation.

5.1 INFLUENCE DE LA PROFONDEUR DE MODULATION. - Envisageons donc un modulateur multiple à $n$ rassembleurs. Nous savons que l'angle de transit jusqu'à la cible, d'un ion de phase d'entrée $\theta_{1}$, est donné par:

$$
\begin{aligned}
\theta_{\mathrm{c} n}=\theta_{1}+ & (n-1) \pi+L_{n}- \\
& -\left[2(n-1) L_{n}+(n-1)^{2} \pi\right] \alpha \sin \theta_{1}
\end{aligned}
$$

expression dans laquelle nous avons négligé les termes du second degré en $\alpha$.

Si nous admettons que la phase d'entrée de la particule centrale est et reste nulle ce qui est une hypothèse raisonnable, nous aurons successivement:

$$
\theta_{\mathrm{cn} 0}=L_{n}+(n-1) \pi
$$

angle de transit de l'ion central

$$
\begin{aligned}
\theta_{\mathrm{c} n_{1}}=\theta_{11}+ & L_{n}+(n-1) \pi- \\
& -\left[2(n-1) L_{n}+(n-1)^{2} \pi\right] \alpha \sin \theta_{11}
\end{aligned}
$$

angle de transit de la particule extrême de l'impulsion d'entrée $\left(\theta_{11}=\Delta \theta_{1}\right.$ : demi-largeur à l'entrée).

La demi-largeur à la cible sera alors donnée par $\Delta \theta_{\mathrm{c} n}=\Delta \theta_{1}-\left[2(n-1) L_{n}+(n-1)^{2} \pi\right] \alpha \sin \Delta \theta_{1}$.

$\mathrm{Si}$ on considère que

$$
\delta \Delta \theta_{\mathrm{c} n} \simeq \frac{\mathrm{d}}{\Delta \theta_{\mathrm{c} n}} \mathrm{~d} \alpha \alpha
$$

nous pourrons écrire

$$
\begin{aligned}
& \delta \Delta \theta_{\mathrm{c} n}= \\
& \quad=-\left[2(n-1) L_{n}+(n-1)^{2} \pi\right] \sin \Delta \theta_{1} \delta \alpha .
\end{aligned}
$$

Remarquons ici que nous considérons que la variation de $\alpha$ est due à une variation de la tension de modulation. La tension $U_{0}$ sous laquelle les ions ont été accélérés reste constante puisque nous considérons que $L_{n}$ est constant. De plus, l'expression de départ éq. (9) implique que l'angle de transit de l'ion de phase d'entrée nulle, dans les différentes cellules, soit bien égal à $\pi$.

Pour évaluer l'expression (10), on peut se contenter d'une valeur approchée de $L_{n}$, dans laquelle on néglige les termes carrés en $\alpha$, c'est-à-dire.

$$
L_{n}=\frac{1-(n-1)^{2} \alpha \pi}{2(n-1) \alpha} \text {. }
$$

En reportant cette valeur dans l'éq. (10), nous avons

$$
\delta \Delta \theta_{\mathrm{cn}}=-\frac{1}{\alpha} \sin \Delta \theta_{1} \delta \alpha
$$

donc

$$
\frac{\delta \Delta \theta_{c n}}{\Delta \theta_{c n}}=-\frac{\sin \Delta \theta_{1}}{\Delta \theta_{c n}} \frac{\delta \alpha}{\alpha}
$$

or, comme

$$
G=\frac{\Delta \theta_{1}}{\Delta \theta_{\mathrm{c} n}}
$$

nous aurons enfin

$$
\frac{\delta G}{G}=\frac{G}{\Delta \theta_{1}} \sin \Delta \theta_{1} \frac{\delta \alpha}{\alpha} .
$$

Il semble donc d'après ce résultat qu'il serait intéressant d'augmenter $\alpha$ afin d'accroître le gain et ce pour une distance de regroupement constante.

Mais dans ces conditions, il apparaît que si $\alpha$ croît au-delà de sa valeur optimum, un phénomène nouveau a lieu.

Ceci est mis en évidence sur le graphique figure 4 : on y voit que certaines particules en rattrapent d'autres parties avant elles. Il en est ainsi par exemple pour la particule de phase d'entrée $\theta_{11}$ qui arrive sur la cible après la particule de phase $\theta_{12}$ partie cependant après elle.

Or, il faut se rappeler que, dans les calculs, nous avons négligé l'effet de charge d'espace. Et, dans cette nouvelle situation, cet effet deviendra vraisemblablement fort important et tendra à s'opposer à ces dépassements de particules au sein d'une même impulsion.

Il serait, par conséquent, peu réaliste de tirer des conclusions générales des relations établies ci-dessus. Cependant, si la largeur d'impulsion à l'entrée est assez grande et telle que la phase d'entrée des particules extrêmes se situe dans une région où $\theta_{c n}$ est une fonction univoque de $\theta_{1}$, et $\mathrm{si}$, de plus, les variations 


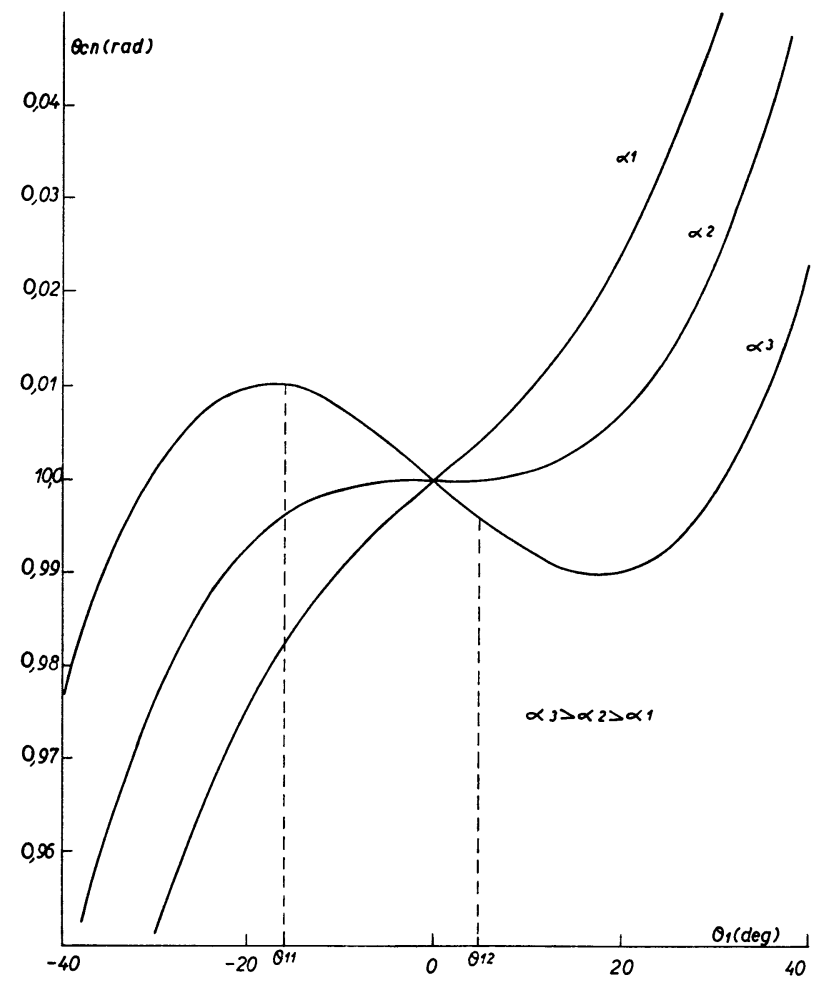

Fig. 4. - Influence d'une variation de $\alpha$ sur l'angle de transit pour une distance totale constante.

de $\alpha$ restent suffisamment petites, hypothèses généralement rencontrées en pratique, on peut alors valablement utiliser la relation (11). C'est en respectant ces deux conditions que le graphique de la figure 5 a été établi. On y a représenté la variation de $G_{\mathrm{T}}$, obtenu comme décrit plus haut, en fonction de $\alpha$ pour une distance totale de regroupement et une largeur d'impulsion d'entrée constantes, et ce pour trois types de modulation. Ce graphique permet de relever la variation relative du gain théorique pour une variation connue de $\alpha$ et de la comparer à la valeur tirée de la formule (11). Le tableau ci-dessous résume les résultats obtenus pour une variation relative de $\alpha$ de $2 \%$.

$$
\begin{array}{ccccc}
n & 3 & 4 & 5 & \text { pour } \Delta \theta_{1}=60^{\circ} \\
- & - & - & - & - \\
\frac{\Delta G_{\mathrm{T}}}{G_{\mathrm{T}}} & 9,5 \% & 10 \% & 10,2 \% & \text { et } \frac{\Delta \alpha}{\alpha}<0
\end{array}
$$$$
\frac{\Delta G}{G} \quad 10,3 \% \quad 10,8 \% \quad 11,4 \%
$$

5.2 INFLUENCE DE LA FRÉQUENCE. - Il est également utile, lors de la réalisation d'un ensemble de regroupement, de savoir quelle est la stabilité en fréquence qu'il faut exiger de l'électronique de modulation, afin de conserver une impulsion ionique de largeur suffisamment reproductible.

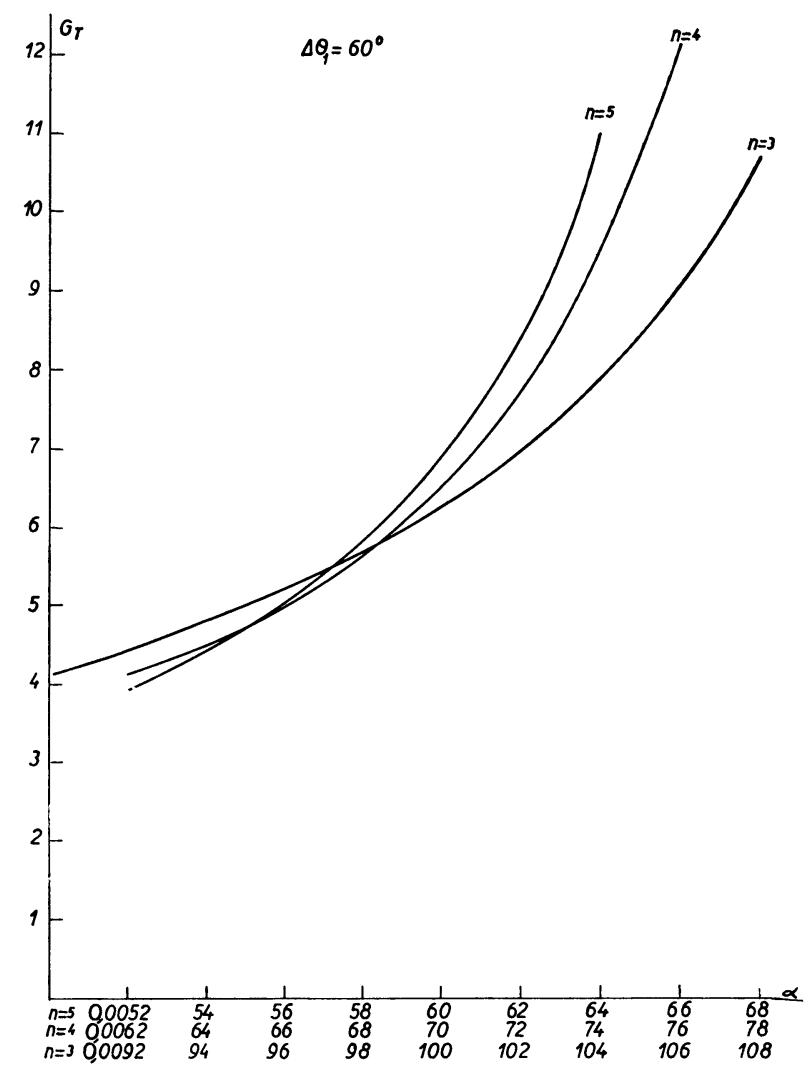

Fig. 5. - Variation du gain théorique avec $\alpha$ pour $L_{n}$ et $\Delta \theta_{1}$ constants.

L'éq. (9) peut s'écrire :

$$
\begin{aligned}
t_{\mathrm{c} n}=t_{1}+ & (n-1) \frac{l_{\mathrm{c}}}{v_{0}}+\frac{l_{n}}{v_{0}}- \\
- & {\left[(n-1) \frac{l_{\mathrm{c}}}{v_{0}}+\frac{2 l_{n}}{v_{0}}\right] \alpha(n-1) \sin \omega t_{1} }
\end{aligned}
$$

où $l_{\mathrm{c}}$ et $l_{n}$ sont respectivement les longueurs des cellules et la longueur de glissement.

En admettant encore que la phase d'entrée de la particule médiane est et reste nulle, cette expression nous conduira à la valeur suivante pour la demilargeur à la cible :

$\Delta t_{\mathrm{c} n}=\Delta t_{1}-\left[(n-1) \frac{l_{\mathrm{c}}}{v_{0}}+\frac{2 l_{n}}{v_{0}}\right] \alpha(n-1) \sin \omega \Delta t_{1}$

et nous aurons

$$
\begin{aligned}
\delta \Delta t_{\mathrm{c} n}=-\left[(n-1) \frac{l_{\mathrm{c}}}{v_{0}}\right. & \left.+\frac{2 l_{n}}{v_{0}}\right] \\
& \times \alpha(n-1) \Delta t_{1} \cos \omega \Delta t_{1} \delta \omega .
\end{aligned}
$$

Comme précédemment, nous remplacerons $l_{n}$ par une valeur approchée négligeant les termes en $\alpha^{2}$, ce qui donne

$$
\frac{\delta \Delta t_{\mathrm{c} n}}{\Delta t_{\mathrm{c} n}}=-\frac{\Delta t_{1} \cos \Delta \theta_{1}}{\omega \Delta t_{\mathrm{c} n}} \delta \omega
$$


et donc

$$
\frac{\delta G}{G}=G \cos \Delta \theta_{1} \frac{\delta \omega}{\omega}
$$

Les courbes de la figure 6, calculées à partir des expressions théoriques de départ, représentent la variation $\mathrm{du}$ gain avec la fréquence pour trois types de modulation.

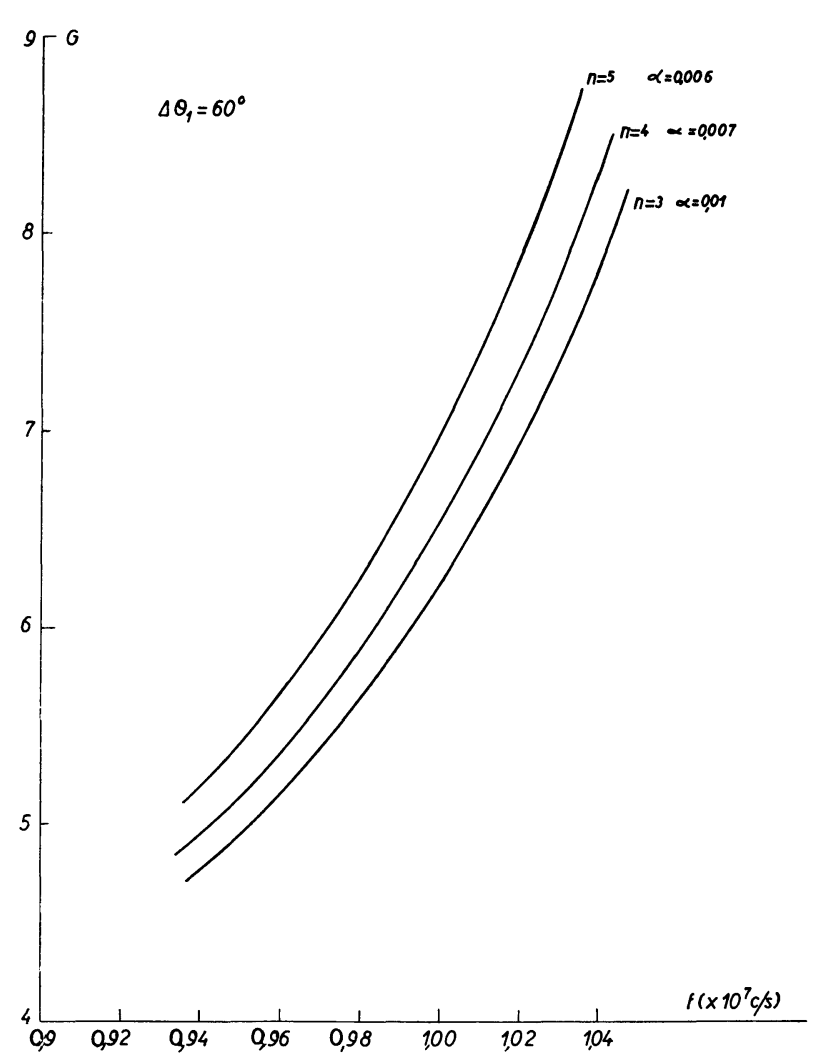

Fig. 6. - Variation du gain avec la fréquence

Remarquons que ces courbes sont tracées pour un intervalle de phase d'entrée constant et non pas pour un intervalle de temps constant. Il est possible de formuler théoriquement ces conditions à l'entrée. Il suffit de réécrire l'éq. (12) et considérer $\Delta \theta_{1}$ comme constant. Nous aurons alors

$$
\Delta t_{\mathrm{c} n}=\frac{\Delta \theta_{1}}{\omega}-\left[(n-1) \frac{l_{\mathrm{c}}}{v_{0}}+\frac{2 l_{n}}{v_{0}}\right] \alpha(n-1) \sin \Delta \theta_{1}
$$

et

$$
\frac{\delta \Delta t_{\mathrm{c} n}}{\Delta t_{\mathrm{c} n}}=-\frac{\Delta \theta_{1}}{\Delta \theta_{\mathrm{c} n}} \frac{\delta \omega}{\omega}
$$

donc

$$
\frac{\delta G}{G}=G \frac{\delta \omega}{\omega}
$$

ainsi pour $n=5, \alpha=6 \times 10^{-3}$ et $\Delta \theta_{1}=60^{\circ}$, la formule (14) donne

$$
\frac{\delta G}{G}=7 \% \quad \text { pour } \quad \frac{\delta \omega}{\omega}=1 \% \text {. }
$$

On retrouve cette variation de $G$ sur le graphique de la figure 6 pour une même variation de la fréquence.

Dans les mêmes conditions de regroupement, une même variation de fréquence $(1 \%$ ) entraînera une variation de moitié du gain si c'est la durée de l'impulsion d'entrée qui est maintenue constante.

On peut s'en assurer en appliquant la formule (13) qui entraîne pour

$$
\begin{aligned}
& \frac{\delta \omega}{\omega}=1 \% \\
& \frac{\delta G}{G}=3,5 \% .
\end{aligned}
$$

Notons cependant que le fait d'avoir utilisé la formule (12) pour déterminer l'influence des variations de fréquence sur le gain restreint les limites d'applicabilité des résultats établis. En effet, nous ne tenons pas compte de la variation de l'angle de transit dans les cellules.

Donc pour que cet angle reste environ égal à $\pi$, on ne peut envisager que de petites variations de fréquence qui se situent cependant dans des limites facilement accessibles à l'expérience. A titre d'exemple, le graphique figure 7 représente la variation de $G$ avec la fréquence dans le cas où on inclut dans le calcul la variation de l'angle de transit dans les cellules de modulation. On voit que, dans ce cas, la dépendance du gain vis-à-vis de la fréquence est fonction du type de modulation.

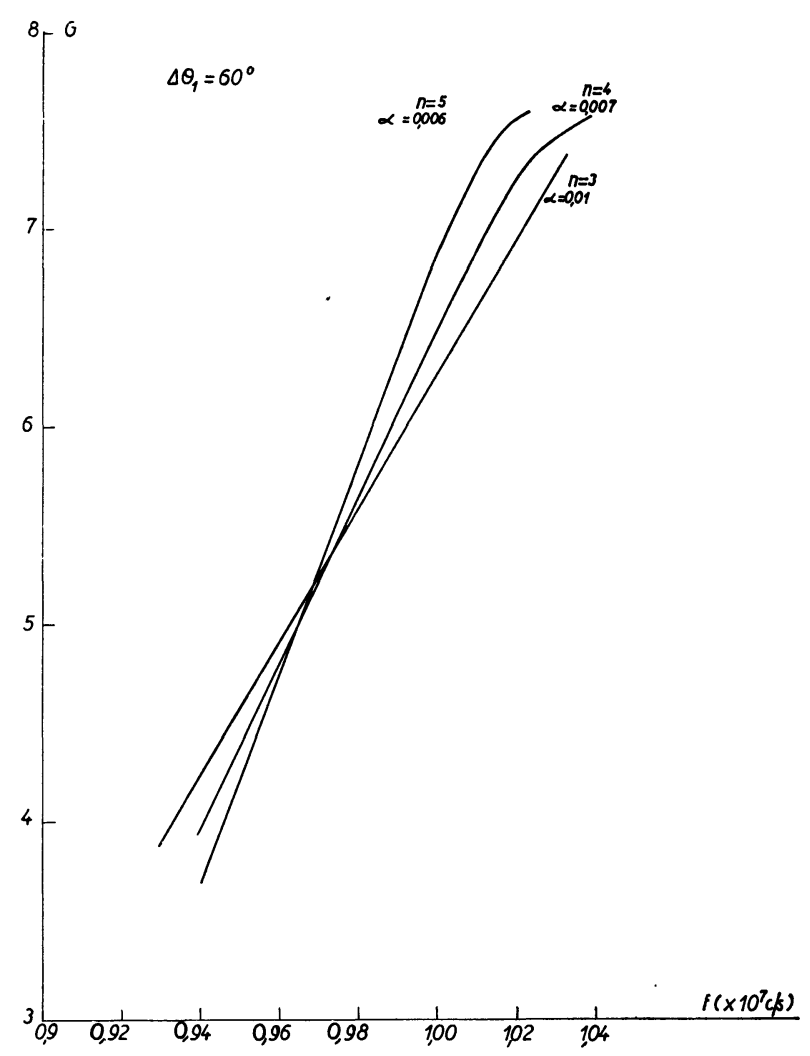

Fig. 7. - Variation du gain avec la fréquence incluant la variation de l'angle de transit dans les cellules. 
Notons cependant que les formules établies ci-dessus (13) et (14) rendent convenablement compte des variations de $G$ pour des écarts de fréquence de l'ordre de $1 \%$.

5.3 INFLUENCE DE LA Vitesse initiale.. - Il est aussi important de connaître la dispersion d'énergie admissible à l'entrée du regroupeur étant donné l'existence d'une distribution d'énergie parmi les particules disponibles à la sortie du pulsateur. De plus, dans le cas de regroupement après accélération, une source supplémentaire de dispersion s'introduit du fait de l'inévitable instabilité de la tension d'accélération.

Pour déterminer l'influence d'une variation de $v_{0}$ sur la largeur d'impulsion ionique, reprenons l'éq. (12) sous la forme :

$$
\begin{aligned}
t_{\mathrm{c} n}= & t_{1}+(n-1) \frac{l_{\mathrm{c}}}{v_{0}}+\frac{l_{n}}{v_{0}}- \\
& -\left[(n-1) \frac{l_{\mathrm{s}}}{v_{0}}+\frac{2 l_{n}}{v_{0}}\right](n-1) \frac{U_{q}}{m v_{0}^{2}} \sin \omega t_{1} .
\end{aligned}
$$

En admettant à nouveau que le regroupement s'effectue autour de l'ion de phase d'entrée nulle, nous pourrons déterminer le temps de transit de la dernière particule participant à l'impulsion

$$
\begin{aligned}
t_{\mathrm{c} n}=\Delta t_{1}+ & (n-1) \frac{l_{\mathrm{c}}}{v_{0}}+\frac{l_{n}}{v_{0}}- \\
& -\left[(n-1) \frac{l_{\mathrm{c}}}{v_{0}}+\frac{2 l_{n}}{v_{0}}\right](n-1) \frac{U_{q}}{m v_{0}^{2}} \sin \Delta \theta_{1}
\end{aligned}
$$

d'où on tire l'expression de la demi-largeur à la cible

$\Delta t_{\mathrm{c} n}=\Delta t_{1}-\left[(n-1) \frac{l_{c}}{v_{0}}+\frac{2 l_{n}}{v_{0}}\right] \times$

$$
\times(n-1) \frac{U_{q}}{m v_{0}^{3}} \sin \Delta \theta_{1}
$$

dans ce cas

$$
\begin{aligned}
\delta \Delta t_{\mathrm{c} n}=\left[(n-1) \frac{l_{\mathrm{c}}}{v_{0}}\right. & \left.+\frac{2 l_{n}}{v_{0}}\right] \\
& \times(n-1) \propto \sin \Delta \theta_{1} 3 \frac{\delta v_{0}}{v_{0}} .
\end{aligned}
$$

Si on remplace dans (16) $l_{n}$ par une valeur approchée, à savoir

$$
l_{n}=\frac{v_{0}}{\omega} \frac{1-(n-1)^{2} \alpha \pi}{2(n-1) \alpha}
$$

nous aurons alors

$$
\frac{\delta \Delta t_{\mathrm{c} n}}{\Delta t_{\mathrm{cn}}}=\frac{3 G}{\Delta \theta_{1}} \sin \Delta \theta_{1} \frac{\delta v_{0}}{v_{0}} .
$$

Il est évident que le domaine de validité de cette expression (17) est restreint dans la même mesure et pour les mêmes raisons que celui des expressions (14) et (15) ci-dessus.

Notons qu'il sera généralement nécessaire de recourir à un tri en énergie du faisceau avant regroupement. En effet, une dispersion d'énergie à l'entrée du regroupeur a une influence assez marquée sur le gain. Pour une impulsion d'entrée de $30 \mathrm{~ns}$ environ à $10 \mathrm{Mc} / \mathrm{s}$, une variation d'énergie de $2 \%$ autour de $250 \mathrm{keV}$ entraîne une variation du gain de l'ordre de $20 \%$.

6. Conclusions. - A l'aide des formules établies ci-dessus, il est possible de déterminer les paramètres caractéristiques d'un modulateur à plusieurs rassembleurs. De plus, les relations liant les variations du gain aux fluctuations possibles des différents paramètres de l'ensemble permettent de déterminer les contraintes mécaniques et électriques à s'imposer afin de disposer à la cible d'impulsions de largeur convenablement reproductibles. Soit, par exemple, à déterminer les caractéristiques d'un modulateur quadruple afin d'obtenir des impulsions cible de neutrons ayant $1 \mathrm{~ns}$ de large. La tension d'accélération est de $250 \mathrm{kV}$, la distance disponible de $2,5 \mathrm{~m}$, on décide d'utiliser une fréquence de $15 \mathrm{Mc} / \mathrm{s}$.

On aura donc

$$
L \text { total }=\frac{\omega l}{v_{0}} \simeq 15 \pi
$$

donc $L_{n}=L_{4}=12 \pi$ ce qui entraîne comme valeur de $\alpha$

$$
\alpha \simeq 4 \times 10^{-3} \quad \text { et } \quad U \simeq 2 \mathrm{kV} .
$$

Il sera alors nécessaire d'alimenter le modulateur avec des impulsions de $15 \mathrm{~ns}$ environ afin de recueillir à la cible des impulsions de 1 ns. Supposons que le regroupeur soit destiné à des expériences de temps de vol. En admettant que la base de vol est de $4 \mathrm{~m}$, le temps moyen que mettront les neutrons de $14 \mathrm{MeV}$ pour la parcourir sera de $80 \mathrm{~ns}$. On pourra donc déterminer le temps de vol de ces neutrons avec une précision de $0,6 \%$ environ si l'incertitude sur l'instant de départ, c'est-à-dire sur la largeur des impulsions cible, est de 0,5 ns ou $50 \%$.

Les résultats acquis ci-dessus nous permettent alors de calculer les fluctuations admissibles sur les différents paramètres du regroupeur :

1) La formule (11) nous obligera à construire un générateur sinusoïdal dont l'amplitude aura une stabilité de $4 \%$ environ.

2) Il ne sera par contre pas nécessaire de prendre des précautions particulières en ce qui concerne la stabilité en fréquence puisque la formule (13) nous indique qu'une fluctuation de $14 \%$ serait admissible.

3) Enfin, la relation (17) nous indique que la tension d'accélération devra être stabilisée à $3 \%$ environ.

Cet ensemble de renseignements permettra à l'expé- 
rimentateur de dimensionner son installation et évitera par exemple de rechercher des performances qui s'avèrent inutiles.

Remerciements. - Nous remercions M. le Profes- seur P. Kipfer, Chef de Service au Laboratoire de Physique Electronique de l'Université Libre de Bruxelles, où ce travail a été réalisé, ainsi que MM. J. Devooght et Sylin pour les conseils et avis qu'ils nous ont donnés.

\section{Bibliographie}

[1] BeCKURTS, J. H., Nucl. Instr. and Methods 11 (1961) 144.

[2] NeILER, J. H., Good, W. M., Fast neutron physics, Vol. 1 (éd. J. B. Marion and J. L. Fowler, Interscience Publ., N. Y.) 1960.

[3] Mobley, R. C., Phys. Rev. 88 (1952) 360.
[4] Roche, M., CEA-R-3796 (1969).

[5] Olivier, C. S., Collince, B., Kaye, G., Nucl. Instr. and Methods 50 (1967) 109.

[6] Ripouteau, F., Thèse de doctorat, Faculté des Sciences de Grenoble (1962). 